\title{
Difficult-to-Treat-Depression and GPs' Role: Perceptions of Psychiatry Registrars
}

\author{
Kay M. Jones, Leon Piterman \\ Office of the Pro Vice-Chancellor, Peninsula Campus, Monash University, Frankston, Australia \\ Email: kay.jones@monash.edu
}

Received 16 July 2014; revised 12 August 2014; accepted 9 September 2014

Copyright (C) 2014 by authors and Scientific Research Publishing Inc.

This work is licensed under the Creative Commons Attribution International License (CC BY).

http://creativecommons.org/licenses/by/4.0/

(c) () Open Access

\begin{abstract}
Introduction: For patients, GPs are the most accessible medical resource in the community and are the gatekeepers to other community resources including psychiatrists. Qualifying as a psychiatrist in Australia involves completing a five-year training program that includes rotations in hospitals and community settings. The aims of this research were to 1) explore psychiatry registrars' perceptions of difficult-to-treat-depression (DTTD) and 2) what they thought about the GPs' role in this regard. Methods: A semi-structured interview schedule comprising six questions was used; 10 psychiatry registrars (6 females, 4 males) participated in a one-and-half-hour focus group. All were in their final year of training and undertaking a training post in a public hospital in Melbourne, Australia. Data were analysed using the Framework Method. Findings: Similar to GPs and GP trainees, psychiatry registrars' perceptions and understanding of DTTD varied. While acknowledging limited experience in diagnosis and management, issues important to them included the utility of labels such as DTTD; patients distressed because of another diagnosis, substance abuse and/or life problems, the importance of accurate histories and notes, cost and limited availability of services particularly in the private sector, prescribing regimens, referring to allied health professionals, and suggesting/prescribing non pharmacological and/or complementary treatment. Also what was of concern was communication, both between health professionals and between health professionals and patients. Consensus was that treating depression in general practice is one of the hardest things for GPs to manage but there was value in using mental health plans. Discussion and Conclusion: While this cohort was small in number with limited experience, this study is the first to contribute to the literature that provides some insight into psychiatry registrars' experiences and perceptions of DTTD. Outcomes may have implications for thepsychiatry training program and GPs who diagnose and manage patients with mental health problems.
\end{abstract}

\section{Keywords}

Depression, GP, Psychiatry

\footnotetext{
${ }^{*}$ Corresponding author.
} 


\section{Introduction}

Against the background of researching GPs [1] and GP trainees' perceptions of difficult-to-treat depression [2] (DTTD), this research was undertaken to explore psychiatry registrars' perceptions of DTTD and what they think about the GPs' role. Since the endorsement of the National Mental Health Strategy in 1992, several initiatives have been introduced, including the Better Access program in 2007 [3]. Among other things, this initiative improved access to psychiatrists [3]. In the 2011-12 year, a conservative estimate of 1348 (full time equivalent) consultant psychiatrists and psychiatrists were employed in state and territory public hospitals, psychiatric units, community care mental care services and residential mental health services in Australia [4]. While the range of reform measures included greater emphasis on care within the community, resulting in an increase of service providers, linkages and communication pathways [5] [6], for some patients, access to psychiatrists remains limited because few psychiatrists work outside major cities and inner regional centres and those practising in the private sector are largely inaccessible due to cost [7].

At the end of 2013, there were 1442 psychiatry trainees at various stages of their training [8]. Qualifying as a psychiatrist in Australia involves joining and completing a five-year training program, which is based around rotations that cover ward rounds and case review in adult, child/adolescent and forensic psychiatry, consultation liaison, and experiences in rural psychiatry, indigenous mental health, psychiatry of old age, addiction, electroconvulsive therapy and psychotherapies [8] [9]. This training program provides the trainees with experiences and links to GPs and community services via their rotations in hospitals (public, remote public, private, not-forprofit hospitals) and community settings (public, private, not-for profit community settings, youth custodial facilities, residential facilities and Aboriginal Community Controlled Health Services) [9].

In Australia, GPs are often the patient's first contact point for health because they are the most accessible medical resource in the community and are the gatekeepers to other community resources [7]. In 2011-12 an estimated $12.1 \%$ of GP encounters were mental health-related with depression the most commonly managed problem by a GP [3]-[5] [10]. While it has been reported that GPs may fail to recognise depression in around half of cases, this degree of non-recognition is understandable because of the many barriers to recognition [11] including somatisation because of its relationship to depression and anxiety [12]. Although GPs can access services in both the private and public health sectors, as it is thought to be better for a person's mental health to treat them in the community rather than in a hospital, mental health services are increasingly provided in the community [13] [14]. However, health services and hospitals deliver their public specialist mental health services differently depending on the local environment and catchment area [15]. In addition, while based on the same set of symptoms, there may be differences in the GPs' and psychiatrists' diagnostic decision-making because of the emphasis accorded to different elements of the history [16]. Thus, partnerships in mental health care, particularly between public and private [17] and improved communication between health professionals and health services is extremely important and helps improve the quality of patient care [18].

While the Diagnostic and Statistical Manual of Mental Disorders, Fifth Edition (DSM V) provides information to diagnose, for example, depressive disorders, major depressive disorder and persistent depressive disorder (dysthymia) [19], no clear definition or information is provided for diagnosing and/or managing DTTD. Therefore, this paper uses the description of DTTD as "most often conceptualized in terms of repeated failures to ameliorate depressive symptoms” [20].

No literature could be found that described psychiatry registrars' perceptions of DTTD and what they think about the GPs' role in managing depression in Australia. Because of the absence of literature, it is important to undertake exploratory work to gain an understanding in this area.

The aims of this research were 1) to explore psychiatry registrars' perceptions of DTTD and 2) what they think about the GPs' role in this regard.

\section{Methods}

\subsection{Sample Recruitment}

A convenience sample was recruited via an email forwarded to registrars undertaking a training post in a public hospital in Melbourne, Australia. When potential participants responded and agreed to participate, they provided their contact details (email) for the purpose of the research team advising time, date and venue for the focus group [21] [22]. 


\subsection{Data Collection and Analysis}

A semi-structured interview schedule comprising six headings which had been previously developed and used when interviewing and/or conducting focus groups with GPs [1] and GP trainees [2] (Table 1).

All data were collected in Melbourne; a focus group was held with the psychiatry registrars (6 females, 4 males) which lasted approximately one-and-a-half-hours, was audio-taped and transcribed verbatim. At the time of the focus group, all participants were in their final year of training and undertaking a training post in a public hospital in Melbourne. No other demographic data were collected.

Data were analysed using the Framework Method [24] to understand participants' perspectives. Data were analysed manually and independently by investigators (KJ and LP). When there was a difference of opinion, the issues were discussed and agreement reached [24]. Findings, including discussion are reported under the interview schedule's six headings. Comments are reported as PR1-10.

Ethics approval to conduct the study was obtained from Monash University Human Research Ethics Committee (MUHREC).

\section{Findings}

Findings are reported under the interview schedule's six sub-headings.

Question 1: Understanding of difficult-to-treat depression (DTTD)?

Similar to GPs [1] and GP trainees [2], psychiatry registrars' (PRs) perceptions and understanding of DTTD varied. One thought of DTTD as "being quite multi-factorial" (PR5), another suggested "it could be quite severe psycho-social stress" (PR6).

"So that's saying whether it's a difficult patient or a difficult depression" (PR3).

"That's right: the subjective experience, the symptoms of the depression would be exactly the same in difficult to treat depression. The difficult to treat part comes from something else. I mean, you know, outside. Which is the treatment or its lack of efficacy" (PR8).

One considered the GPs' role:

"I'm not sure [of the meaning of DTTD], but what might be important to GPs is looking at what makes it difficult to treat. I mean rather than maybe defining if it's this cluster of symptoms" (PR5).

One suggested that DTTD may be misdiagnosed:

"That the whole construct of depression has really been flawed and is invalid and non-viable and non-reproducible. There's no really well defined approach on the definition of depression. So difficult to treat depression is really just over-diagnosed non-depression that's been diagnosed as depression and doesn't respond to antidepressants" (PR1).

Question 2: What is your understanding of other terms?

Registrars' understanding of other terms, viz treatment resistant depression, treatment-refractory depression, treatment-resistant major depressive disorder and major depressive disorder varied with around half indicating they had limited understanding of the terms:

"I think we've got an enormous problem with semantics" (PR2).

Table 1. Interview schedule.

1) What is your understanding of the term difficult-to-treat-depression (DTTD)?

2) What is your understanding of other terms; viz: treatment-resistant depression treatment-refractory depression, treatment-resistant major depressive disorder and major depressive disorder?

3) What are your experiences of diagnosing DTTD?

4) What are your experiences of managing DTTD?

5) Does your management of these patients include:

a) using an illness management model or a chronic illness [23];

b) communication with GPs;

c) referring the patient to allied health professionals, and

d) suggesting/prescribing non-pharmacological and/or complementary treatment?

6) If "no" to any of Question 5, have you ever considered using any of these options/other comments? 
"For me, I see difficult to treat as where simple things like some psychiatrist trying antidepressants and CBT don't work. So there's definition by exclusions. And treatment resistant would be the next level. So you have second and third opinions that are multiple antidepressants, mood stabilisers, ECT and these things aren't working" (PR3).

"I still don't know what the difference is between treatment resistant and difficult to treat, or is there a difference, I don't know” (PR4).

"Yes, resistant is a strong word. It's heading more towards incurable in a way, whereas difficult-to-treat-it's a phrase laden with opportunity" (PR8).

The role of the GP and their knowledge of their patients were also discussed. The majority felt the GPs play a significant role in managing patients diagnosed with DTTD:

"I guess GPs who know people and build up that relationship and understanding of their life, their paths, their psycho-social stressors, and the way that the relationship, the way they relate" (PR6).

"I find that if I have been asked to see somebody by another member of the medical team and then I call the GP, the GP can often very clearly say look, they've [the patient] been like this for a long time, this is why this is happening. So I do think GPs have an understanding but I don't know if the system supports them in those areas" (PR5).

\section{Question 3: What are your experiences of diagnosing DTTD?}

While registrars' experience in diagnosing was limited compared to GPs, the registrars all agreed that GPs carry the majority of the burden of mental illness. All agreed that when diagnosing DTTD, the "diagnosis is always loaded with management" (PR9), and the utility of labels to describe an illness or management has positive and negative aspects:

"There are a lot of imprecise things in medicine, I feel we get bogged down getting the exact diagnosis or correct term, and maybe we just need to accept there is a degree of uncertainty and we are going to get our definitions wrong” (PR4).

"We've all [medical practitioners] signed up, psychiatry included, to this evidence based model of medicine and to have evidence you need labels; unfortunately with psychiatry, labelling reduces the infinite human complexity to a circumscribed area, and that circumscribed area is going to have boundaries, and the boundary is necessarily going to be arbitrary” (PR8).

Only one registrar raised using DSM to assist with diagnosis:

“Most psychiatrists don't vigorously use the DSM to make the diagnosis" (PR1).

Much of the discussion focussed on the role and input of the GP:

"I think particularly in general practice there's an enormous amount of good kind of non-billed for counselling/containing of people, because GPs know them for years and understand actually what it is that works and what it is that helps and that's a therapeutic relationship; perhaps it doesn't necessarily matter if it's your GP or your psychiatrist or psychiatry registrar, it's a therapeutic relationship" (PR2).

"I agree, it's the GPs that carry the majority of the burden of mental illness, I think the GPs provide a very holistic human relationship, like a long term human relationship that's vital in the treatment of depression" (PR6).

"We think of GPs generally as holistic practitioners. Some are extremely biological, as you have to acknowledge, but some of them are holistic" (PR7).

Question 4: What are your experiences of managing DTTD?

The registrars reported that in their experience, patients are a very heterogeneous group, some with other mental health disorders and others with substance abuse and/or life problems:

"Trying not to call something treatment resistant or treatment refractory, and also acknowledging that depression that is difficult to treat perhaps encompasses more than mere treatment resistance as defined by DSM depression" (PR10).

"Some of them [patient] have undiagnosed bipolar, lots of personality disorder, substance abuse, existential life problems. I mean it's very hard, all you know is it's basically a kind of 'heart-sink' patient, with low numbers of therapeutic optimism" (PR1).

Another felt that the depression may be difficult to manage because "it isn't what we think that it is" (PR2).

Regarding taking histories and making notes as part of management, all agreed that it is important to take an accurate developmental history:

"It is important to ensure that a developmental history is taken, rather than the psychiatrist saying 'I have not 
had time to take a developmental history'” (PR1).

"Yes, the developmental history gets, you know, delegated to some other time" (PR4).

Management differences were also noted for patients in the public and private sector:

"I'm in outpatients clinic at the moment and being psych registrars in a public hospital we're the second line already, so perhaps you could say that all those that we're seeing depression, it's complicated in some way" (PR5).

While cost and availability were factors in the private sector, there was general consensus that the public sector is not really set up for these patients (PR4), "which can result in these patients not getting a lot of treatment within the public system" (PR1).

"So there is some utility in some of these labels from the perspective of accounting, planning, policy budgetary level" (PR10).

There was agreement that some patients presenting in the outpatient public sector may be distressed because of another diagnosis but aren't necessarily depressed, subsequently:

"You've got to find the right person. I mean, I think it's really quite difficult to do those things, although I'd like to think that I do address those. But I have to say it's much easier to consider medication first" (PR5).

Medication was described as part of management for most patients. Prescribing regiments were variously described:

"A friend of mine was a private psychiatrist, leaves a prescription pad at the reception desk, and it vastly reduces prescribing because she has to go outside to get it" (PR2).

"If I am treating someone with DTTD, I am more likely to perhaps follow an intense medication path if there's a risk... it is important to try as many medications as possible...” (PR6).

“... but not just reach for a prescribing pad”(PR1).

Question 5: Does your management of these patients include

a) using an illness management model [23];

b) communication with GPs;

c) referring to allied health professionals, and/or

d) suggesting/prescribing non pharmacological and/or complementary treatment?

1) Using an illness management model

Similar to the GP trainees, most of the psychiatry registrars were unsure what an illness management model was, one suggested:

"In the system that we work it becomes very much a medical model, you know" (PR4).

\section{2) Communication with GPs}

As psychiatry registrars who are in training posts in public hospitals work in teams, they communicate with a range of health professionals. They described the GPs' involvement with the patient's management as important:

"If it's looking for connectedness, then GPs are often actually a really good person for that. So that could be the GP... who gives them a call every week and listens to them on the phone. You know, if that could be the pathway if that we had mental health nurses that do the check-in all the time, that's the connectedness and that can help" (PR5).

"The GPs know the patients and within that relationship, understand the patient's life and stressors, the way they relate. I think we should also reflect on treatment resistant depression as a sociological problem in that it's the structure of our current modern day society in the west. And these GPs are; they're called upon to be, like a sort of connectedness to the community, and it must be so hard for them because they're so busy and they mean that for a lot of patients" (PR6).

Communication between health professionals was also raised, particularly when a GP refers a patient to a psychiatrist:

"Even if we don't agree with the definitions or they're not clear, we still need a quick way to communicate with our colleagues, and that's what they do in medicine; sometimes the diagnosis might not fit, but it's just a quick way to communicate if it gets through" (PR4).

"If a GP refers someone to you and says they are depressed, you come back with the answer, well what is depression?” (PR8).

\section{3) Referring to allied health professionals}

Whilst the majority had referred to allied health professionals, the majority thought that referring to allied health professionals would depend on the severity (PR1) and complexity of the patient's illness (PR2), and 
whether it will assist to preserve the patient's job and marriage (PR3) rather than the illness becoming their identity (PR6).

"As well as social psychologists, we use other allied health professionals to manage these patients" (PR9).

"I think it comes down to resources, so if it's somebody that's really complex with a lot of risk, and can afford it, probably a psychiatrist. If it's somebody that's quite sever with a lot of risks, probably community mental health or GP” (PR5).

\section{4) Suggesting/prescribing non pharmacological and/or complementary treatment}

Opinion varied; some felt these options may be problematic for a number of reasons, whereas others felt there is an increasing evidence base, thus some options have sufficient value to be considered as part of the management plan:

"I think there is increasing evidence base for the variety of complementary medicines with which to treat depression and I think that we as professionals its incumbent upon us to be knowledgeable about them. I'm involved with a mob of GPs that use integrative medicine as a way of treating the patient so using the best evidence from complementary alternative therapies and from mainstream for treating somebody with depression... and there are some GPs who are extremely well trained in that, or some GPs who know very good naturopaths, or other complementary medical practitioners who they can refer to" (PR2).

"I'm thinking thinks like Mindfulness, yoga" (PR4).

"I went to St John's Wort" (PR9).

"Going back to whether you would have access to a good GP, you know, someone who is willing to spend the time to be broad minded to alternative treatments, all those things, you need a good GP” (PR7).

"And that's rarer than winning gold, a good GP!" (PR2).

Question 6: If no to any of Question 5, have you ever considered using any of those options/other comments?

Participants felt that treating depression in general practice is one of the hardest things for GPs to manage, for example, time-pressure with the patient (PR7) and the patient expecting something concrete (PR7). The use of mental health plans (PR9) and resources such as education (PR2), papers published in journals (PR5) and guidelines (PR9, PR10) were described as important and valuable:

"I think it's very hard for GPs, I think that if there could be a lot less sort of prescribing of SSRIs and a lot more capacity for GPs to feel confident and skilled to look at other ways in which to help manage mild to moderate is one thing about depression. Then that would be great" (PR2).

\section{Discussion and Conclusion}

In response to the various questions, these registrars articulated their perceptions of DTTD and their thoughts about the GPs' role and relationship with the patient generally and with patients who experience depression that is difficult to treat [1] [2].

Rotations into various sectors of the mental health services provided this cohort of registrars with a range of experiences; all had rotated in the public sector, but few had in the private sector [8] [9]. These rotations provided insight into management differences both within the sectors, the various health professionals, and the impact on patients, but also highlighted gaps in the registrars' knowledge and experience. While Commonwealth government initiatives improved access to psychiatrists [3], major factors remain that impact on patients including cost, availability and access, because of limited personal resources and/or Medicare funding requirements [3]-[7].

General consensus was that GPs carry the majority of the burden of diagnosing, managing and treating patients with mental illness [3]-[7]. All had considered community options as part of their management plans; which included a range of treatment options including GPs, allied health professionals and complementary and alternative treatments [1] [2]. From the registrars' perspectives, the most important aspect of management was clear, effective communication regardless of whether it was between professionals or between professionals and patients, but as this did not always occur; possible changes to processes were discussed [11] [12] [15]-[18].

Few had referred to DSM V [19] or heard of a chronic disease management model [23], rather, significant emphasis was placed on the GPs' role and input, which was described as important, particularly for the patient [1] [7] [11] [12].

The generalisability of this study may be limited because of the small number of participants who were all 
from metropolitan Melbourne, with no input from the rural sector. Regardless, this study is the first to contribute to the literature by providing some insight into psychiatry registrars' experiences and perceptions of DTTD and what they think about the GPs' role in managing patients diagnosed with DTTD.

\section{Implications for General Practice and Psychiatrist Registrar Training}

- With the increasing number of trainee registrars [9], an increase in the number and/or duration of rotations to rural and remote rural locations would provide support in areas that have limited healthcare professionals.

- To enhance communication between health care professionals, discussion on team based care models and mental health care planning processes could be included in the training program, for example in the "consultation liaison" component [9].

- Where possible, GPs support community based rotations by having a trainee registrar in their practice as part of the psychiatry registrar rotation, to better understand each other's roles.

- Where relevant, analysis and report of trainee registrars' direct contact with GPs and allied health professionals, the role of DSM in training registrars to assist with diagnosis may need review.

\section{References}

[1] Jones, K.M., Castle, D.J. and Piterman, L. (2012) Difficult-to-Treat-Depression: What Do General Practitioners Think? MJA Open, 1, 6-8.

[2] Jones, K.M., Piterman, L. and Spike, N. (2014) Difficult-to-Treat-Depression-Perceptions of GPs and GP Trainees. Open Journal of Psychiatry, 4, 228-237. http://dx.doi.org/10.4236/ojpsych.2014.43029

[3] Department of Health and Ageing (2013) National Mental Health Report 2013: Tracking Progress of Mental Health Reform in Australia 1993-2011. Commonwealth of Australia, Canberra.

[4] AIHW: Mental Health Services in Australia (2012) Staffing of State and Territory Specialised Mental Health Care Facilities. https://mhsa.aihw.gov.au/resources/facilities/staffing/

[5] Private Mental Health Alliance (PMHA) (2011) Principles for Collaboration, Communication and Cooperation between Private Mental Health Service Providers. Private Mental Health Alliance, Kingston.

[6] Pirkis, J., Harris, M., Hall, W. and Ftanou, M. (2011) Evaluation of the Better Access to Psychiatrists, Psychologists and General Practitioners through the Medicare Benefits Schedule Initiative. Summative Evaluation, Final Report. Centre for Health Policy, Programs and Economics, The University of Melbourne.

[7] Senate Select Committee Mental Health (2006) A National Approach to Mental Health—From Crisis to Community. Final Report. Senate Select Committee, Commonwealth of Australia, Canberra.

[8] Royal Australian and New Zealand College of Psychiatrists (RANZCP) (2014) Quarterly Education Activities Report January-December 2013. RANZCP, Melbourne.

[9] Royal Australian and New Zealand College of Psychiatrists (RANZCP) (2013) Education Activities Report for the 2012 Training Year. RANZCP, Melbourne.

[10] AIHW (2013) Mental Health Services-In Brief 2013 Cat.no.HSE 141. AIHW, Canberra.

[11] Clarke, D.M., Cook, K., Smith, G.C. and Piterman, L. (2008) What Do General Practitioners Think Depression Is? A Taxonomy of Distress and Depression for General Practitioners. MJA, 188, S110-S113.

[12] Clarke, D.M., Piterman, L., Byrne, C.J. and Austin, D.W. (2008) Somatiac Symptoms, Hypochondriasis and Psychological Distress: A Study of Somatisation in Australian General Practice. MJA, 189, 560-564.

[13] Better Health: The Roles of Psychiatrists and General Practitioners. http://www.betterhealth.vic.gov.au/bhcv2/bhcarticles.nsf/pages/Mental illness treatments

[14] Keks, N.A., Altson, M., Sacks, T.L., Hustig, H.H. and Tangahow, A. (1997) Collaboration between General Practice and Commkunity Psychiatric Services for People with Chronic Mental Illness. The Medical Journal of Australia, 167, 266-271.

[15] Victorian Government (2011) Victorian Government Health Information: Adult Specialist Mental Health Services (16 - 64 Years). http://www.betterhealth.vic.gov.au/bhcv2/bhcarticles.nsf/pages/Mental_illness_treatments

[16] Lampe, L., Fritz, K., Boyce, P., Starcevic, V., Brakoulias, V., Walter, G., Shadbolt, N. and Harris, A. and Malhi, G. (2013) Psychiatrists and GPs: Diagnostic Decision Making, Personality Profiles and Attitudes toward Depression and Anxiety. Australasian Psychiatry, 21, 231-237. http://dx.doi.org/10.1177/1039856213486210

[17] Yung, A., Gill, L., Somerville, E., Dowling, B., Simon, K., Pirkis, J., Livingston, J., Schweitzer, I., Tanaghow, A., 
Herrman, H., et al. (2005) Public and Private Psychiatry: Can They Work Together and Is It Worth the Effort? Australian and New Zealand Journal of Psychiatry, 39, 67-73. http://dx.doi.org/10.1080/j.1440-1614.2005.01511.x

[18] Sain, K., Shah, N. and Hasan, S. (2012) GPs’ Views on the Content and Quality of Psychiatrists' Clinic Letters. Progress in Neurology and Psychiatry, 16, 6-10. http://dx.doi.org/10.1002/pnp.223

[19] American Psychiatric Association (2013) Diagnostic and Statistical Manual of Mental Disorders. 5th Edition (DSM-5), American Psychiatraic Publishing, Washington DC.

[20] Grote, N.K. and Frank, E. (2003) Difficult-to-Treat Depression: The Role of Contexts and Comorbidities. Society of Biological Psychiatry, 53, 660-670. http://dx.doi.org/10.1016/S0006-3223(03)00006-4

[21] Liamputtong, P. and Ezzey, D. (2005) Qualitative Research Methods. Oxford University Press, Melbourne.

[22] Polgar, S. and Thomas, S. (2005) Introduction to Research in the Health Sciences. Elsevier Churchill Livingstone, Sydney.

[23] Wagner, E.H. (1998) Chronic Disease Management: What Will It Take to Improve Care for Chronic Illness? Effective Clinical Practice, 1, 2-4.

[24] Ritchie, J. and Spencer, L. (1994) Qualitative Data Analysis for Applied Policy Research. In: Bryman, A. and Burgess, B., Eds., Analyzing Qualitative Data, Routledge, London, Chapter 9. http://dx.doi.org/10.4324/9780203413081 
Scientific Research Publishing (SCIRP) is one of the largest Open Access journal publishers. It is currently publishing more than 200 open access, online, peer-reviewed journals covering a wide range of academic disciplines. SCIRP serves the worldwide academic communities and contributes to the progress and application of science with its publication.

Other selected journals from SCIRP are listed as below. Submit your manuscript to us via either submit@scirp.org or Online Submission Portal.
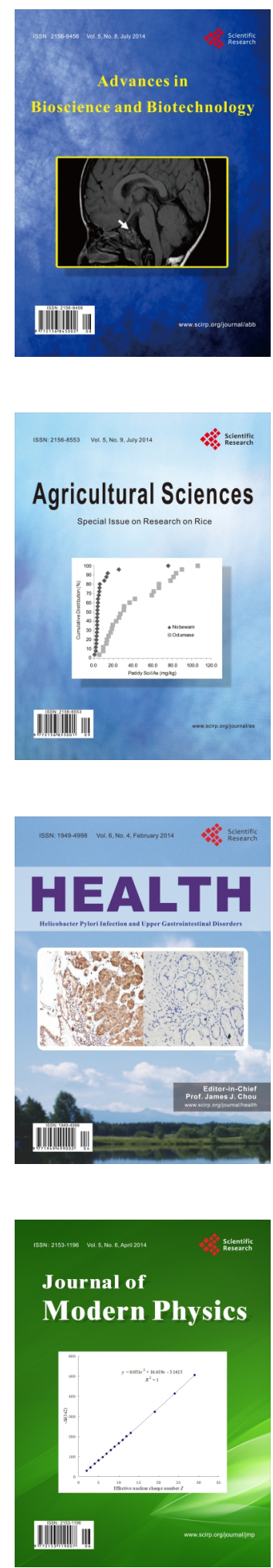
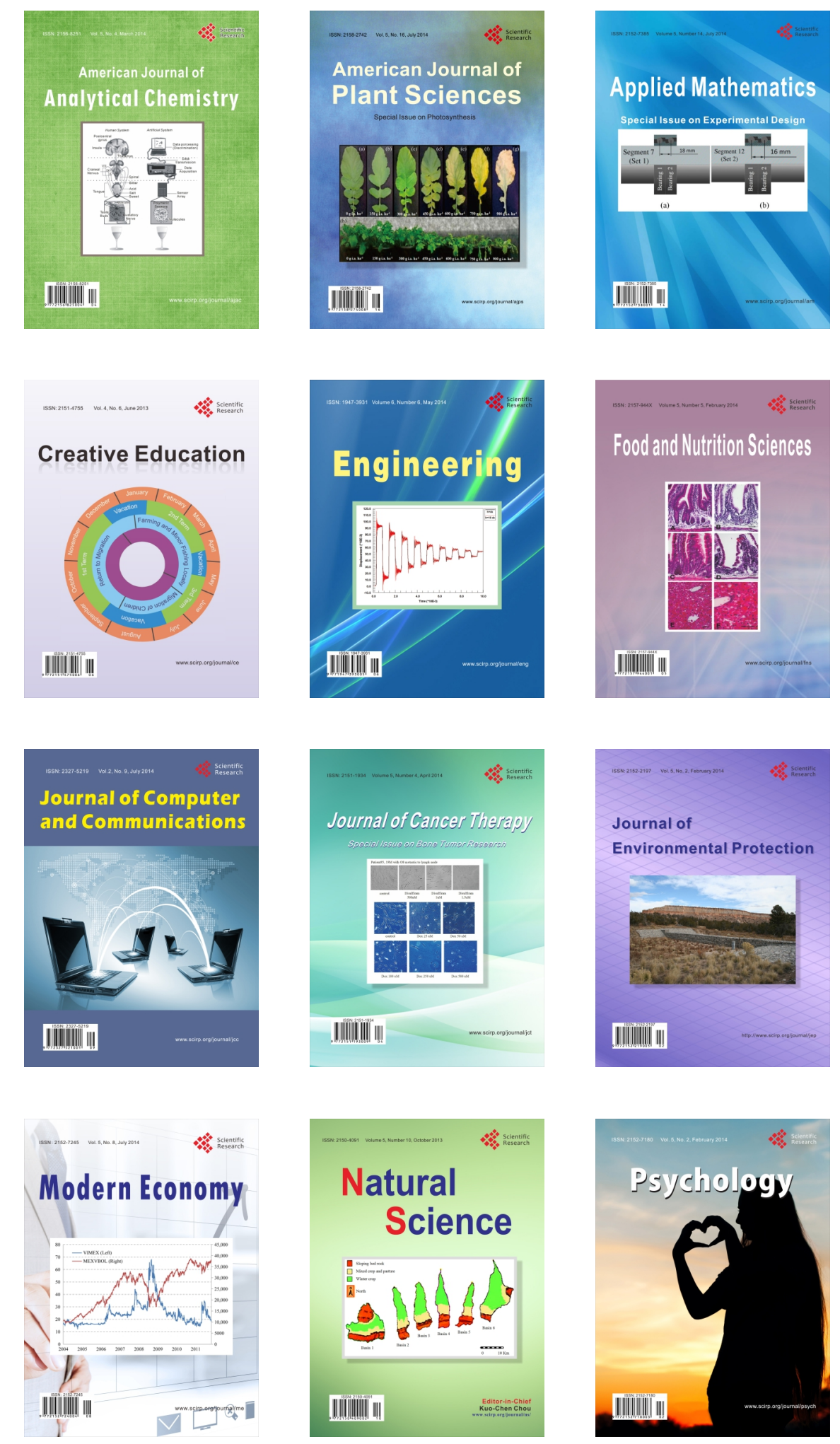